An electron beam detector for the FLASH II beam dump

This article has been downloaded from IOPscience. Please scroll down to see the full text article.

2013 J. Phys.: Conf. Ser. 425122012

(http://iopscience.iop.org/1742-6596/425/12/122012)

View the table of contents for this issue, or go to the journal homepage for more

Download details:

IP Address: 131.169.44.34

The article was downloaded on 10/04/2013 at 13:30

Please note that terms and conditions apply. 


\title{
An electron beam detector for the FLASH II beam dump
}

\author{
J Good $^{2}$, G Kube ${ }^{1}$, N Leuschner ${ }^{2}$, F Perlick ${ }^{2 *}$, M Sachwitz ${ }^{2}$, M Schmitz ${ }^{1}$, K \\ Wittenburg $^{1}$ and $T$ Wohlenberg ${ }^{1}$ \\ ${ }^{1}$ DESY Hamburg site, Notkestraße 85, 22607 Hamburg, Germany \\ ${ }^{2}$ DESY Zeuthen site, Platanenallee 6, 15738 Zeuthen, Germany \\ *E-mail: florian.perlick@desy.de
}

\begin{abstract}
After the generation of the laser light, a dipole deflects the highly energetic electron beam of the „Free Electron Laser Hamburg“ into a dump. To control the position, dimensions and profile of the electron beam and to avoid contact with adjacent components, causing a total breakdown of the linac, a detector is developed. Light is emitted due to the electrons hitting a luminescent screen, and is then reflected by a mirror, located in $2 \mathrm{~m}$ distance from the screen, and passes through a vacuum window to a CCD camera. An experimental setup is currently built, representing quite closely the terms of installation at FLASH II. In this setup the ceramics $\mathrm{Al}_{2} \mathrm{O}_{3}$ and Chromox will be examined as screen materials.
\end{abstract}

\section{Introduction}

The Free Electron Laser Hamburg (FLASH) is a linear accelerator producing brilliant laser light from 4 to $60 \mathrm{~nm}$ wavelengths, providing unique experimental opportunities to investigate the atomic structure and the properties of materials, nanoparticles, viruses and cells. After the generation of the laser light in the undulator sections the electrons are separated from the FEL beam. While the laser light is guided to the experimental sites in the experimental hall, a dipole deflects the electrons downwards at an angle of $27^{\circ}$ to dispose of them into a dump (see figure 1).

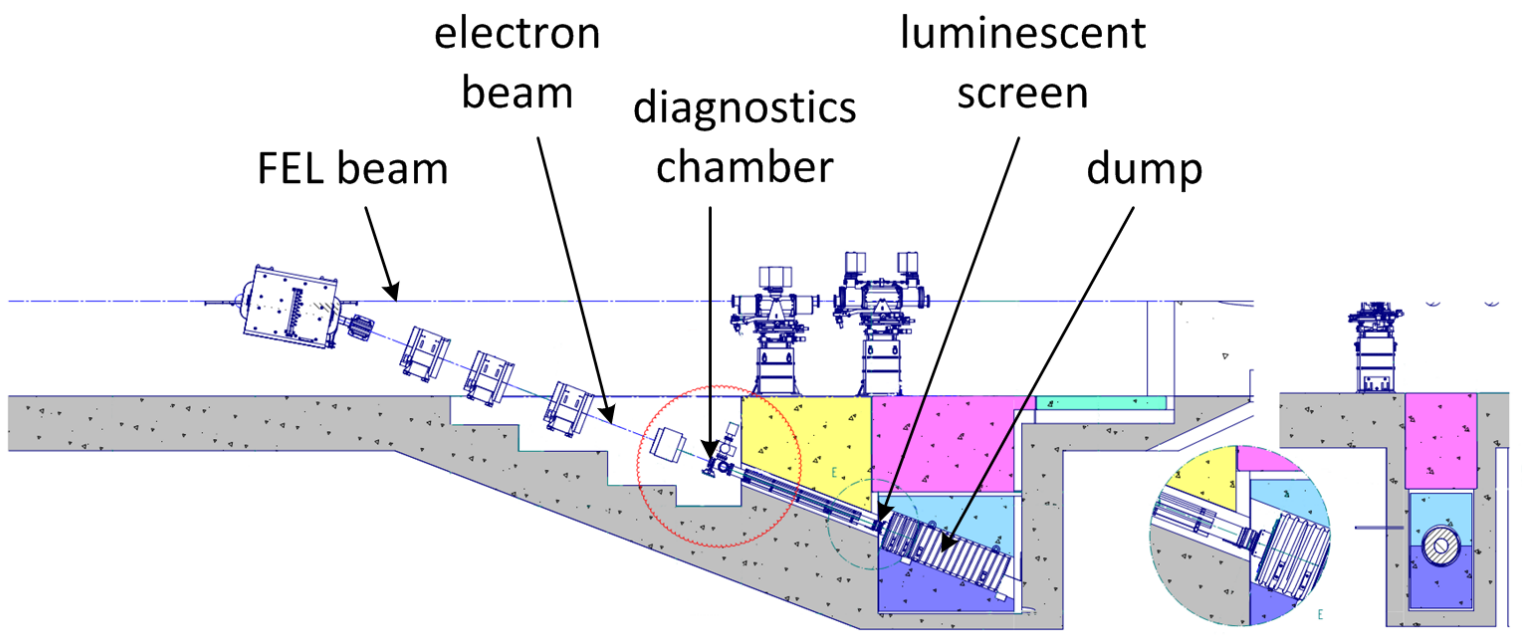

Figure 1. Beam dump section at FLASH II 
The high power beam is able to easily damage beam line components in the case of misdirection. Therefore, a detector is required at the dump to control the shape and the position of the beam. The detector will be surveyed continuously by the accelerator control room to immediately detect any beam optic failures. External coupling is not permitted due to the almost impossible access/maintenance at the termination of the beam pipe. The detector has to be able to operate and survive in a highly radioactive environment. Therefore a luminescent screen, $100 \mathrm{~mm}$ diameter in size, is installed in front of the dump aperture. At a diagnostics chamber, installed approximately in $2 \mathrm{~m}$ distance from the screen, the emitted light is reflected by a mirror through a vacuum window to a CCD camera. Since the camera is one of the components which have to be well protected from radiation, it is placed about one and a half meters away from the beam line, shielded by the concrete, at ground level. For guiding the light to the camera two different techniques will be applied redundantly. In addition to a conventional lens-mirror-system, radiation-hard optical fibres will be used. The entire optical system is supposed to have a resolution better than $1 \mathrm{~mm}$. During the operation of FLASH II the beam is expected in the middle of the screen. Beam misdirection towards the screen edges will first lead to an alarm signal, and if the beam is outside the screen where it is invisible for the CCD, FLASH will be shut down immediately to avoid potential damage.

\section{Experimental setup}

In order to optimize the parameters of the screen, the mirror, the lens system and the camera, a test setup is currently built in the lab, representing quite closely the terms of installation at FLASH II. Comparatively to the accelerator, the distance between the luminescent screen and the mirror is about $2 \mathrm{~m}$. In the lab the electron beam is created by an electron gun which is installed behind the screen. Although its maximum beam energy $(5 \mathrm{KeV})$ is far below the energy of the FLASH electron beam (1 $\mathrm{GeV}$ ), the electrical charge is comparable. FLASH has an electrical charge of about $1 \mathrm{nC}$, a value which can be achieved with the experimental setup by combining a continuous electron gun filament current of $10 \mu \mathrm{A}$ with a camera shutter speed of $0.1 \mathrm{~ms}$.

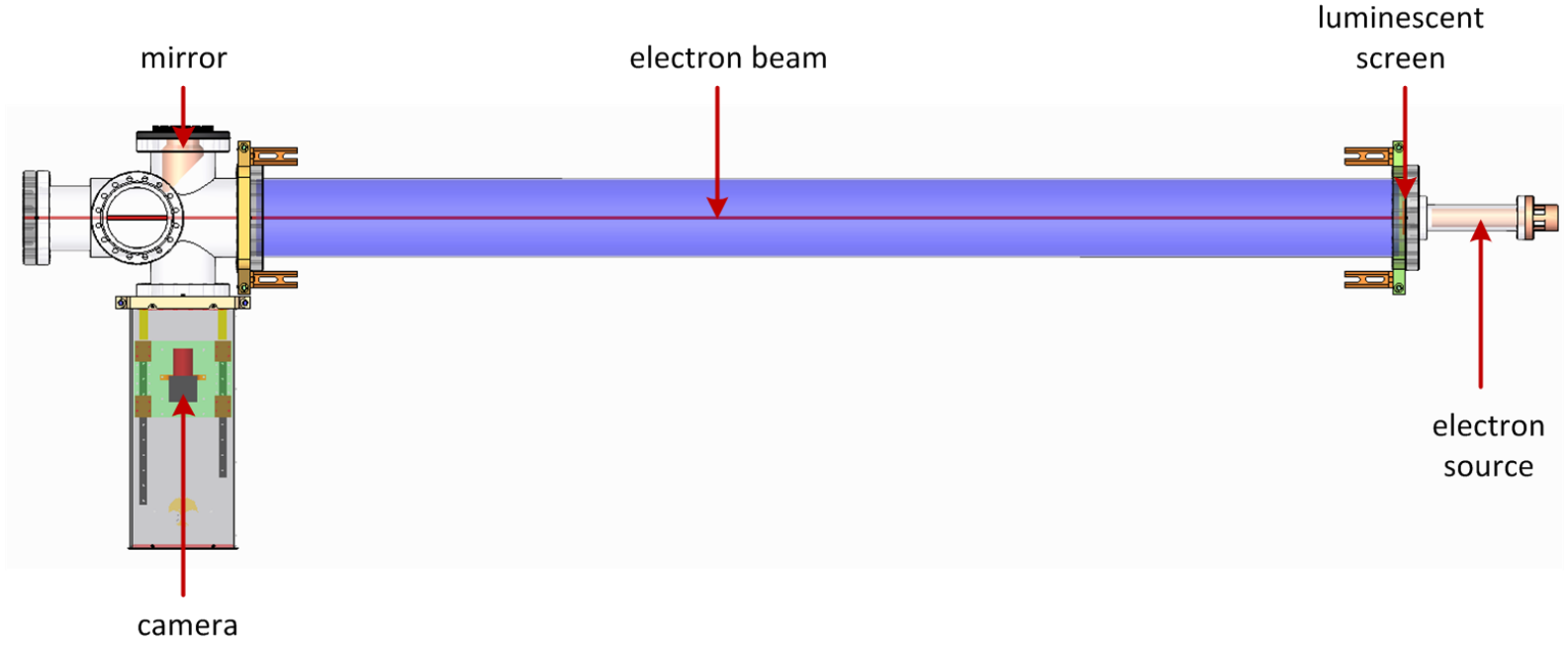

Figure 2. Experimental setup

The mirror (see figure 5) extends $20 \mathrm{~mm}$ into the beam line and reflects the light emitted at the rear side of the luminescent screen. The mirror is made of Glidcop AL15, a sintered alloy consisting of copper and particles of aluminium oxide. Glidcop combines high strength with good thermal conductivity. The reflecting surface is lapped, polished and manufactured at an angle of $46.1^{\circ}$ to the electron beam to guide the light to the centre of the lens. The mirror is soldered directly onto the vacuum flange. Gold foil is used as brazing solder in order to assure best possible transfer of the heat generated by the beam halo. 


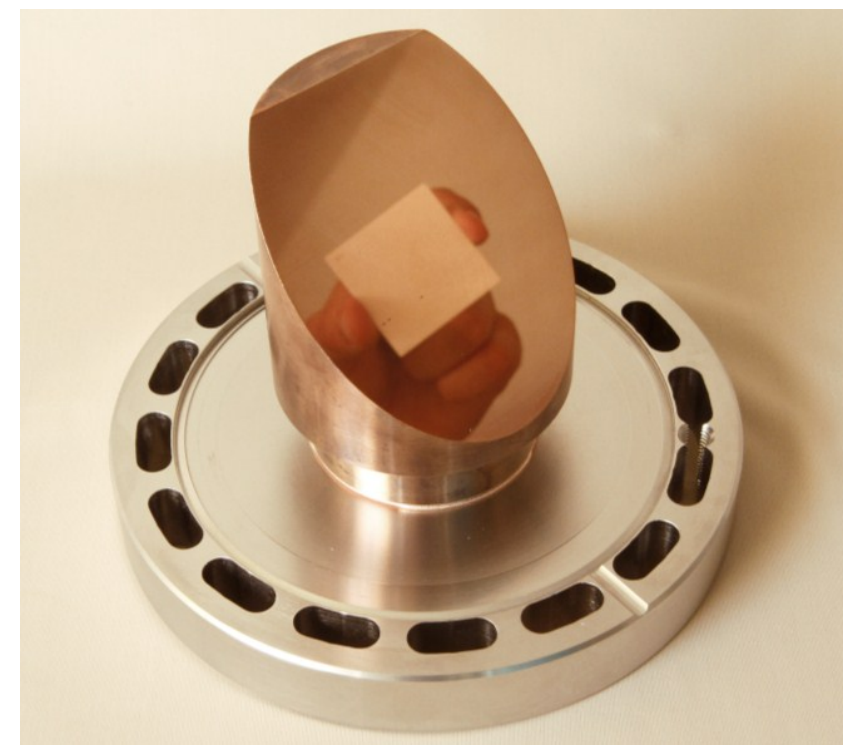

Figure 3. Glidcop mirror

\section{Estimation of mirror temperature due to heat input}

Since the possibility cannot be ignored that electrons of the beam halo may hit the mirror, a thermal simulation was made to get a rough estimate of the resulting mirror temperatures. At FLASH the average energy input into a material per beam train is about $1.2 * 10^{20} \mathrm{eV} / \mathrm{g}^{*} \mathrm{~cm}^{-2}$. According to the installation conditions of the mirror the maximum depth of impression into the Glidcop (density ca. $8900 \mathrm{~g} / \mathrm{cm}^{3}$ ) is approximately $1.5 \mathrm{~cm}$. Considering the FLASH frequency of $10 \mathrm{~Hz}$ the resulting thermal power the mirror has to deal with can be up to $2570 \mathrm{~W}$.

To optimize the heat transfer from the flange to the environment, cooling fins were machined into it. For the simulation, the boundary conditions at the mirror illustrate a worst case scenario. The heat transfer to the diagnostics chamber is restricted, so the flange is the only part where convection is possible (see figure 4). The estimated film coefficient of $5 \mathrm{~W} / \mathrm{m}^{2} * \mathrm{~K}$ represents restrained convection, i.e. due to stagnant air and a flange contaminated with settled dust. The ambient temperature in the dump region was presumed to be $30^{\circ} \mathrm{C}$.

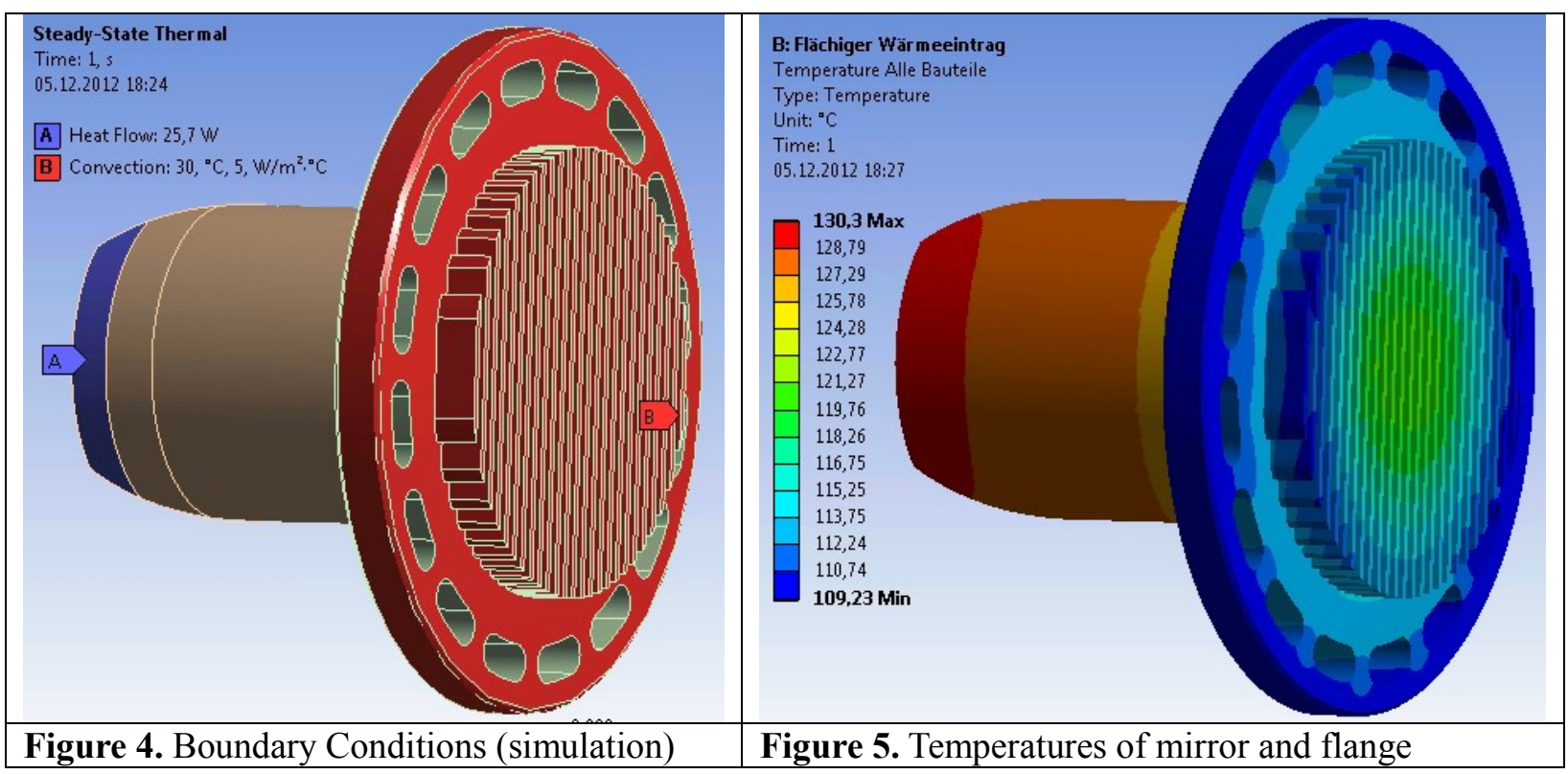


For the first simulation, only $1 \%$ of the possible thermal beam power $(25.7 \mathrm{~W})$ is applied evenly to the mirror on the blue surface (see figure 4). The mirror reaches up to $130^{\circ} \mathrm{C}$, while the maximum temperature of the flange is about $110^{\circ} \mathrm{C}$ (see figure 5). Another simulation, where the beam hits the mirror at a small selected point at the top of the mirror (1 mm diameter), shows that around the point of impact the mirror reaches a temperature of up to $212^{\circ} \mathrm{C}$, while the overall temperatures of the mirror and the flange are similar to the previous simulation. The last simulation, where $10 \%$ of the thermal beam power is applied to the Glidcop, results in a maximum temperature of $1030^{\circ} \mathrm{C}$ at the top of the mirror and $820^{\circ} \mathrm{C}$ at the outer face of the flange. These temperatures are far too high, so that in this case the cooling system has to be significantly improved.

\section{Initial tests in the lab}

For initial tests, the light was reflected directly towards a CCD camera which was made displaceable by linear bearings, allowing the testing of various camera-lens-combinations. Two different ceramics, $\mathrm{Al}_{2} \mathrm{O}_{3}$ and $\mathrm{Al}_{2} \mathrm{O}_{3}: \mathrm{Cr}$ (Chromox), each of them with a thickness of $1 \mathrm{~mm}$, were tested. It could be seen that the Chromox screen $(0.639$ photons $/ \mathrm{keV})$ yields almost twice as much light as the one made of $\mathrm{Al}_{2} \mathrm{O}_{3}(0.367$ photons $/ \mathrm{keV})$ [1]. Degrading of the screen material will be tested to select the optimal component for FLASH II.

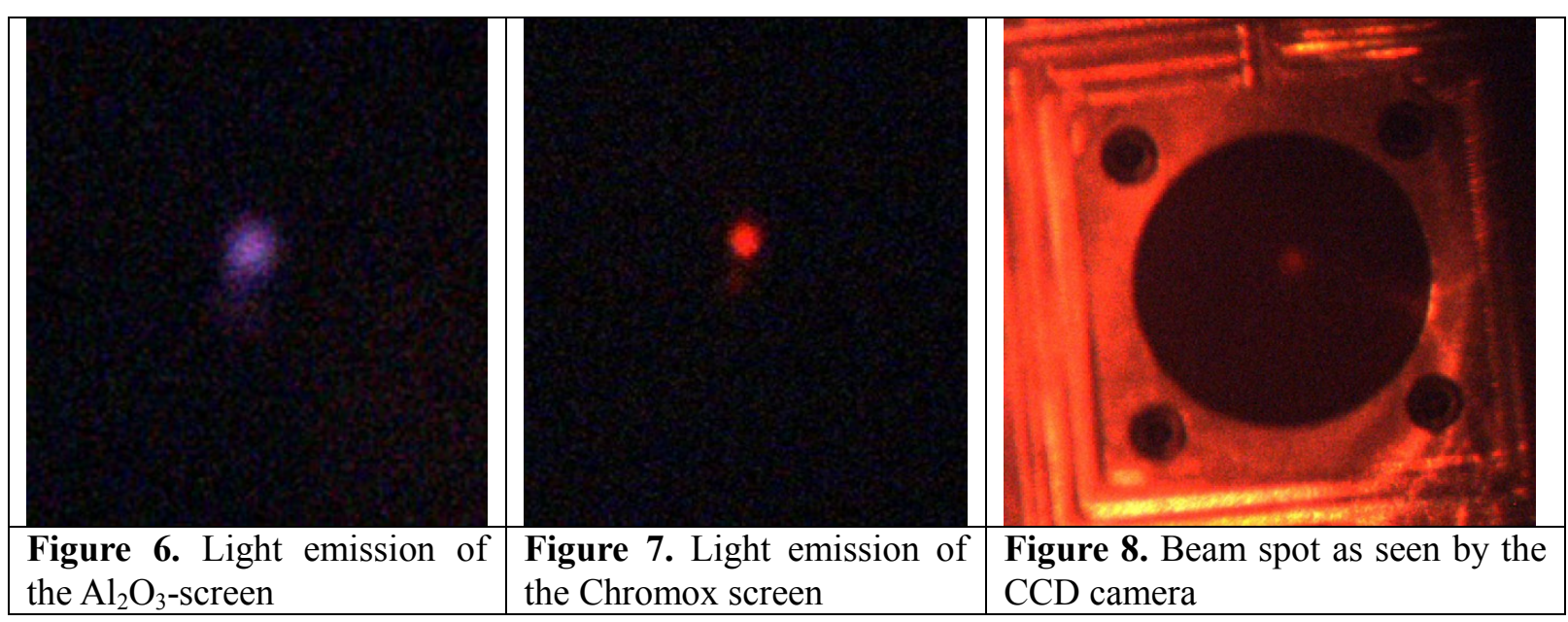

\section{Outlook}

The experimental setup shown in figure 1, which has already been built up in the lab, will be completed by the light guiding system. Both the lens-mirror-system and the optical fibres will be tested in advance, supplemented by a test of possible radiation damage to the fibres which shall be placed onto a "radioactive hot spot" at the bunch compressor in the FLASH accelerator. Finally, radiation protection shall be developed for lenses and electronic parts to counteract the degradation of their optical qualities.

\section{References}

[1] Kube G and Lauth W 2009 Investigation of the light yield of luminescent screens for high energy and high brilliant electron beams proceedings of DIPAC09, Basel, Switzerland, TUPD39 